SRNL-STI-2012-00698

Revision 0

Keywords: $M C U, A R P, I S D P$

Retention: Permanent

\title{
Results of Analysis of Macrobatch 3 Decontaminated Salt Solution Coalescer from May 2010
}

T. B. Peters

S. D. Fink

December 2012

Savannah River National Laboratory Savannah River Nuclear Solutions Aiken, SC 29808

Prepared for the U.S. Department of Energy under

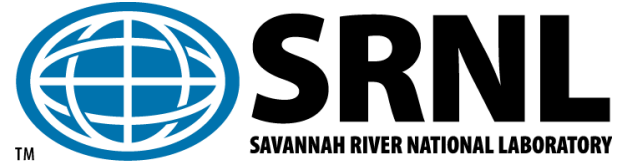
contract number DE-AC09-08SR22470. 
SRNL-STI-2012-00698

Revision 0

\section{DISCLAIMER}

This work was prepared under an agreement with and funded by the U.S. Government. Neither the U.S. Government or its employees, nor any of its contractors, subcontractors or their employees, makes any express or implied:

1. warranty or assumes any legal liability for the accuracy, completeness, or for the use or results of such use of any information, product, or process disclosed; or

2. representation that such use or results of such use would not infringe privately owned rights; or

3. endorsement or recommendation of any specifically identified commercial product, process, or service.

Any views and opinions of authors expressed in this work do not necessarily state or reflect those of the United States Government, or its contractors, or subcontractors.

\section{Printed in the United States of America}

Prepared for

U.S. Department of Energy 


\section{REVIEWS AND APPROVALS}

\section{AUTHORS:}

T. B. Peters, Author, SRNL/SASP

Date

TECHNICAL REVIEW:

C. A. Nash, Technical Reviewer, SRNL/ACP

Date

APPROVAL:

S. D. Fink, SRNL/SASP, Manager

Date

S. L. Marra, SRNL/E\&CPT Research Programs, Manager

Date

D. J. Martin, H Tank Farm Process Engineering

Date 


\section{EXECUTIVE SUMMARY}

SRNL analyzed the Decontamination Salt Solution (DSS) coalescer from MCU by several analytical methods. This unit was removed from service in May 2010. The results of these analyses indicate that there is very little evidence of fouling via excessive solids, either from the leaching studies or X-Ray Diffraction (XRD) analysis. 


\title{
LIST OF ABBREVIATIONS
}

\author{
ARP - Actinide Removal Process \\ DSS - Decontaminated Salt Solution \\ ICPES - inductively-coupled plasma emission spectroscopy \\ MCU - Modular Caustic-Side Solvent Extraction Unit \\ MST - monosodium titanate \\ PSID - pounds per square inch differential \\ SE - Strip Effluent \\ SRNL - Savannah River National Laboratory \\ XRD - X-ray diffraction
}




\subsection{Introduction}

The Decontaminated Salt Solution (DSS) coalescer is used to reduce the organic (mainly Isopar ${ }^{\circledR} \mathrm{L}$ ) carryover into the aqueous phase. The coalescer is made of Ryton ${ }^{\circledR}$ (polyphenylene sulfide). The DSS coalescer has a nominal 20 micron particle removal rating - which means that if used as a filter, the unit will reject $99.98 \%$ of all particles 20 microns or larger. During operations with Macrobatches 1 and 2, both types of coalescers at times exhibited pressure drops in excess of the $<10$ psid expected from non-radiological testing. ${ }^{1}$ In May 2010, during the processing of Macrobatch 3, the DSS coalescer exhibited signs of excessive fouling (20-30 psi pressure drop). The coalescer was removed from service and sent to the Savannah River National Laboratory (SRNL) for analysis. The coalescer was drained of liquids in the facility before removal but no significant effort was made to chemical clean the elements.

\subsection{Experimental Procedure}

When the item arrived at SRNL, the researchers performed a visual study to look for obvious defects or plugging.

Personnel then cut 1" wide rings from the coalescer element. These rings were each completely immersed in $100 \mathrm{~mL}$ of $0.85 \mathrm{M}$ or $3 \mathrm{M}$ nitric acid or $5 \mathrm{M} \mathrm{NaOH}$ for $\sim 1$ month at room temperature. No agitation was applied other than when the sample was initially immersed. Samples from the leachates were removed at 1, 7, 14, and 28 days. The leachate samples were analyzed by Inductively Coupled Plasma Emission Spectroscopy (ICPES).

Samples of unleached coalescer material were also analyzed by X-ray diffraction (XRD).

Details for the work are contained in a controlled laboratory notebook. ${ }^{2}$

\subsection{Results and Discussion}

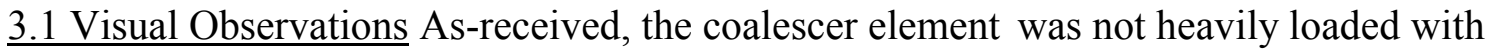
solids and had minimal discoloration. No loose solids or free liquids were associated with this element.

3.2 Leaching Studies Samples from the leaching studies were analyzed by ICPES (see Tables 1-3). The analytical uncertainty for the ICPES samples is $10 \%$. 
Table 1. 0.85M HNO $\mathrm{HCPES}_{3}$ Results for the Coalescer Element

\begin{tabular}{|c|c|c|c|c|c|}
\hline \multirow{2}{*}{ Analyte } & \multicolumn{4}{|c|}{ ICPES Sample Results (mg/L) } & \multirow{2}{*}{$\begin{array}{c}\text { Source Materia } \\
(\mathrm{mg} / \mathrm{L})\end{array}$} \\
\hline & 1 hour & 24 hours & 336 hours & 672 hours & \\
\hline $\mathrm{Ag}$ & $<0.214$ & $<0.214$ & $<0.214$ & $<0.214$ & $<2.14$ \\
\hline $\mathrm{Al}$ & 7.03 & 7.17 & 7.27 & 7.08 & 5290 \\
\hline $\mathrm{B}$ & $<0.375$ & $<0.375$ & $<0.375$ & $<0.375$ & 82.6 \\
\hline $\mathrm{Ba}$ & $<0.118$ & $<0.118$ & $<0.118$ & $<0.118$ & $<0.59$ \\
\hline $\mathrm{Be}$ & $<0.011$ & $<0.011$ & $<0.011$ & $<0.011$ & $<0.7$ \\
\hline $\mathrm{Ca}$ & 0.806 & 0.81 & 0.867 & 0.802 & 2.27 \\
\hline $\mathrm{Cd}$ & $<0.035$ & $<0.035$ & $<0.035$ & $<0.035$ & 1.17 \\
\hline $\mathrm{Ce}$ & $<0.66$ & $<0.66$ & $<0.66$ & $<0.66$ & $<6.6$ \\
\hline $\mathrm{Co}$ & $<0.085$ & $<0.085$ & $<0.085$ & $<0.085$ & NA \\
\hline $\mathrm{Cr}$ & 0.176 & 0.178 & 0.212 & 0.198 & 71.6 \\
\hline $\mathrm{Cu}$ & $<0.142$ & $<0.142$ & $<0.142$ & $<0.142$ & 1.68 \\
\hline $\mathrm{Fe}$ & 0.874 & 1.11 & 1.28 & 1.31 & 12.2 \\
\hline $\mathrm{Gd}$ & $<0.136$ & $<0.136$ & $<0.136$ & $<0.136$ & $<2.12$ \\
\hline $\mathrm{K}$ & $<2$ & $<2$ & $<2$ & $<2$ & 480 \\
\hline $\mathrm{La}$ & $<0.108$ & $<0.108$ & $<0.108$ & $<0.108$ & $<1$ \\
\hline $\mathrm{Li}$ & $<0.756$ & $<0.756$ & $<0.756$ & $<0.756$ & 25.6 \\
\hline $\mathrm{Mg}$ & 1.26 & 1.29 & 1.34 & 1.32 & $<0.25$ \\
\hline $\mathrm{Mn}$ & 0.038 & 0.0455 & 0.041 & 0.043 & 0.88 \\
\hline Mo & $<0.427$ & $<0.427$ & $<0.427$ & $<0.427$ & 8.47 \\
\hline $\mathrm{Na}$ & 225 & 228 & 228 & 221 & 157000 \\
\hline $\mathrm{Nb}$ & $<0.115$ & $<0.115$ & $<0.115$ & $<0.115$ & NA \\
\hline $\mathrm{Ni}$ & 0.711 & 0.739 & 0.739 & 0.74 & $<2.35$ \\
\hline $\mathrm{P}$ & 2.22 & 3.17 & 3.63 & 3.71 & 272 \\
\hline $\mathrm{Pb}$ & $<0.731$ & $<0.731$ & $<0.731$ & $<0.731$ & $<7.31$ \\
\hline $\mathrm{S}$ & $<7.5$ & $<7.5$ & $<7.5$ & $<7.5$ & 2410 \\
\hline $\mathrm{Sb}$ & $<0.688$ & $<0.688$ & $<0.688$ & $<0.688$ & $<10.4$ \\
\hline $\mathrm{Si}$ & 0.746 & 0.88 & 0.862 & 0.946 & 168 \\
\hline $\mathrm{Sn}$ & $<0.429$ & $<0.429$ & $<0.429$ & $<0.429$ & $<4.29$ \\
\hline $\mathrm{Sr}$ & $<0.008$ & $<0.008$ & $<0.008$ & $<0.008$ & $<0.08$ \\
\hline Th & $<0.891$ & $<0.891$ & $<0.891$ & $<0.891$ & NA \\
\hline $\mathrm{Ti}$ & 8.1 & 10.6 & 11 & 10.7 & $<0.17$ \\
\hline $\mathrm{U}$ & $<4.45$ & $<4.45$ & $<4.45$ & $<4.45$ & $<44.5$ \\
\hline $\mathrm{V}$ & $<0.052$ & $<0.052$ & $<0.052$ & $<0.052$ & $<0.52$ \\
\hline $\mathrm{Zn}$ & 0.872 & 0.9 & 0.9 & 0.895 & 6.5 \\
\hline $\mathrm{Zr}$ & $<0.047$ & $<0.047$ & $<0.047$ & $<0.047$ & $<0.89$ \\
\hline
\end{tabular}

$\mathrm{NA}=$ not available. 
Table 2. 3M HNO $\mathrm{HCPES}_{3}$ Results for the Coalescer Element

\begin{tabular}{|c|c|c|c|c|c|}
\hline \multirow{2}{*}{ Analyte } & \multicolumn{4}{|c|}{ ICPES Sample Results (mg/L) } & \multirow{2}{*}{$\begin{array}{c}\text { Source Material } \\
(\mathrm{mg} / \mathrm{L})\end{array}$} \\
\hline & 1 hour & 24 hours & 336 hours & 672 hours & \\
\hline $\mathrm{Ag}$ & $<0.214$ & $<0.214$ & $<0.214$ & $<0.214$ & $<2.14$ \\
\hline $\mathrm{Al}$ & 9.89 & 9.96 & 9.76 & 9.94 & 5290 \\
\hline $\mathrm{B}$ & $<0.375$ & $<0.375$ & $<0.375$ & $<0.375$ & 82.6 \\
\hline $\mathrm{Ba}$ & $<0.118$ & $<0.118$ & $<0.118$ & $<0.118$ & $<0.59$ \\
\hline $\mathrm{Be}$ & $<0.011$ & $<0.011$ & $<0.011$ & $<0.011$ & $<0.7$ \\
\hline $\mathrm{Ca}$ & 1.42 & 1.42 & 1.44 & 1.43 & 2.27 \\
\hline $\mathrm{Cd}$ & $<0.035$ & $<0.035$ & $<0.035$ & $<0.035$ & 1.17 \\
\hline $\mathrm{Ce}$ & $<0.66$ & $<0.66$ & $<0.66$ & $<0.66$ & $<6.6$ \\
\hline $\mathrm{Co}$ & $<0.085$ & $<0.085$ & $<0.085$ & $<0.085$ & NA \\
\hline $\mathrm{Cr}$ & 0.256 & 0.267 & 0.292 & 0.3 & 71.6 \\
\hline $\mathrm{Cu}$ & $<0.142$ & $<0.142$ & $<0.142$ & $<0.142$ & 1.68 \\
\hline $\mathrm{Fe}$ & 1.75 & 1.99 & 2.26 & 2.36 & 12.2 \\
\hline $\mathrm{Gd}$ & $<0.136$ & $<0.136$ & $<0.136$ & $<0.136$ & $<2.12$ \\
\hline $\mathrm{K}$ & $<2$ & $<2$ & $<2$ & $<2$ & 480 \\
\hline $\mathrm{La}$ & $<0.108$ & $<0.108$ & $<0.108$ & $<0.108$ & $<1$ \\
\hline $\mathrm{Li}$ & $<0.756$ & $<0.756$ & $<0.756$ & $<0.756$ & 25.6 \\
\hline $\mathrm{Mg}$ & 2.06 & 2.09 & 2.15 & 2.17 & $<0.25$ \\
\hline $\mathrm{Mn}$ & 0.0605 & 0.0675 & 0.074 & 0.0725 & 0.88 \\
\hline Mo & $<0.427$ & $<0.427$ & $<0.427$ & $<0.427$ & 8.47 \\
\hline $\mathrm{Na}$ & 301 & 302 & 302 & 302 & 157000 \\
\hline $\mathrm{Nb}$ & $<0.115$ & $<0.115$ & $<0.115$ & $<0.115$ & NA \\
\hline $\mathrm{Ni}$ & 1.24 & 1.29 & 1.29 & 1.29 & $<2.35$ \\
\hline $\mathrm{P}$ & 2.78 & 3.58 & 3.94 & 3.91 & 272 \\
\hline $\mathrm{Pb}$ & $<0.731$ & $<0.731$ & $<0.731$ & $<0.731$ & $<7.31$ \\
\hline $\mathrm{S}$ & $<7.5$ & $<7.5$ & $<7.5$ & $<7.5$ & 2410 \\
\hline $\mathrm{Sb}$ & $<0.688$ & $<0.688$ & $<0.688$ & $<0.688$ & $<10.4$ \\
\hline $\mathrm{Si}$ & 1.3 & 1.29 & 1.39 & 1.41 & 168 \\
\hline $\mathrm{Sn}$ & $<0.429$ & $<0.429$ & $<0.429$ & $<0.429$ & $<4.29$ \\
\hline $\mathrm{Sr}$ & $<0.008$ & $<0.008$ & $<0.008$ & $<0.008$ & $<0.08$ \\
\hline Th & $<0.891$ & $<0.891$ & $<0.891$ & $<0.891$ & NA \\
\hline $\mathrm{Ti}$ & 19.1 & 19.9 & 20.1 & 20.1 & $<0.17$ \\
\hline $\mathrm{U}$ & $<4.45$ & $<4.45$ & $<4.45$ & $<4.45$ & $<44.5$ \\
\hline $\mathrm{V}$ & $<0.052$ & $<0.052$ & $<0.052$ & $<0.052$ & $<0.52$ \\
\hline $\mathrm{Zn}$ & 1.36 & 1.38 & 1.39 & 1.39 & 6.5 \\
\hline $\mathrm{Zr}$ & $<0.047$ & $<0.047$ & $<0.047$ & $<0.047$ & $<0.89$ \\
\hline
\end{tabular}

$\mathrm{NA}=$ not available. 
SRNL-STI-2012-00698

Revision 0

Table 3. 5 M NaOH ICPES Results for the Coalescer Element

\begin{tabular}{|c|c|c|c|c|c|}
\hline \multirow{2}{*}{ Analyte } & \multicolumn{4}{|c|}{ ICPES Sample Results (mg/L) } & \multirow{2}{*}{$\begin{array}{c}\text { Source Material } \\
(\mathrm{mg} / \mathrm{L})\end{array}$} \\
\hline & 1 hour & 24 hours & 336 hours & 672 hours & \\
\hline $\mathrm{Ag}$ & $<2.14$ & $<2.14$ & $<2.14$ & $<2.14$ & $<2.14$ \\
\hline Al & $<9.42$ & $<9.42$ & $<9.42$ & $<9.42$ & 5290 \\
\hline $\mathrm{B}$ & $<3.75$ & $<3.75$ & $<3.75$ & $<3.75$ & 82.6 \\
\hline $\mathrm{Ba}$ & $<1.18$ & $<1.18$ & $<1.18$ & $<1.18$ & $<0.59$ \\
\hline $\mathrm{Be}$ & $<0.11$ & $<0.11$ & $<0.11$ & $<0.11$ & $<0.7$ \\
\hline $\mathrm{Ca}$ & $<0.88$ & $<0.88$ & $<0.88$ & $<0.88$ & 2.27 \\
\hline $\mathrm{Cd}$ & $<0.35$ & $<0.35$ & $<0.35$ & $<0.35$ & 1.17 \\
\hline $\mathrm{Ce}$ & $<6.6$ & $<6.6$ & $<6.6$ & $<6.6$ & $<6.6$ \\
\hline $\mathrm{Co}$ & $<0.85$ & $<0.85$ & $<0.85$ & $<0.85$ & NA \\
\hline $\mathrm{Cr}$ & $<1.05$ & $<1.05$ & $<1.05$ & $<1.05$ & 71.6 \\
\hline $\mathrm{Cu}$ & $<1.42$ & $<1.42$ & $<1.42$ & $<1.42$ & 1.68 \\
\hline $\mathrm{Fe}$ & $<1.05$ & $<1.05$ & $<1.05$ & $<1.05$ & 12.2 \\
\hline $\mathrm{Gd}$ & $<1.36$ & $<1.36$ & $<1.36$ & $<1.36$ & $<2.12$ \\
\hline $\mathrm{K}$ & $<20$ & $<20$ & $<20$ & $<20$ & 480 \\
\hline $\mathrm{La}$ & $<1.08$ & $<1.08$ & $<1.08$ & $<1.08$ & $<1$ \\
\hline $\mathrm{Li}$ & $<7.56$ & $<7.56$ & $<7.56$ & $<7.56$ & 25.6 \\
\hline $\mathrm{Mg}$ & $<0.25$ & $<0.25$ & $<0.25$ & $<0.25$ & $<0.25$ \\
\hline $\mathrm{Mn}$ & $<0.2$ & $<0.2$ & $<0.2$ & $<0.2$ & 0.88 \\
\hline Mo & $<4.27$ & $<4.27$ & $<4.27$ & $<4.27$ & 8.47 \\
\hline $\mathrm{Na}$ & 110000 & 110000 & 110000 & 110000 & 157000 \\
\hline $\mathrm{Nb}$ & $<1.15$ & $<1.15$ & $<1.15$ & $<1.15$ & NA \\
\hline $\mathrm{Ni}$ & $<2.35$ & $<2.35$ & $<2.35$ & $<2.35$ & $<2.35$ \\
\hline $\mathrm{P}$ & $<8.49$ & $<8.49$ & $<8.49$ & $<8.49$ & 272 \\
\hline $\mathrm{Pb}$ & $<7.31$ & $<7.31$ & $<7.31$ & $<7.31$ & $<7.31$ \\
\hline $\mathrm{S}$ & $<75$ & $<75$ & $<75$ & $<75$ & 2410 \\
\hline $\mathrm{Sb}$ & $<6.88$ & $<6.88$ & $<6.88$ & $<6.88$ & $<10.4$ \\
\hline $\mathrm{Si}$ & $<2.52$ & $<2.52$ & $<2.52$ & $<2.52$ & 168 \\
\hline $\mathrm{Sn}$ & $<4.29$ & $<4.29$ & $<4.29$ & $<4.29$ & $<4.29$ \\
\hline $\mathrm{Sr}$ & $<0.08$ & $<0.08$ & $<0.08$ & $<0.08$ & $<0.08$ \\
\hline Th & $<8.91$ & $<8.91$ & $<8.91$ & $<8.91$ & NA \\
\hline $\mathrm{Ti}$ & $<1.1$ & $<1.1$ & $<1.1$ & $<1.1$ & $<0.17$ \\
\hline $\mathrm{U}$ & $<44.5$ & $<44.5$ & $<44.5$ & $<44.5$ & $<44.5$ \\
\hline $\mathrm{V}$ & $<0.52$ & $<0.52$ & $<0.52$ & $<0.52$ & $<0.52$ \\
\hline $\mathrm{Zn}$ & 0.87 & 0.87 & 0.87 & 0.87 & 6.5 \\
\hline $\mathrm{Zr}$ & $<0.47$ & $<0.47$ & $<0.47$ & $<0.47$ & $<0.89$ \\
\hline
\end{tabular}

$\mathrm{NA}=$ not available. 
The source material values are the calculated feed values from Macrobatch $3 .^{3}$ Values marked "NA" in the source materials are analytes that were not available.

A number of trends appear in the leaching data.

First, the use of $5 \mathrm{M} \mathrm{NaOH}$ leads to the ICPES being swamped by the sodium content. No conclusions can be drawn from the $5 \mathrm{M} \mathrm{NaOH}$ leaching, and this data will not be further discussed.

Second, the various analytes in the leachates (Al, B, Cr, Fe, Na, etc) do not possess the same relative concentrations as present in the source material. This indicates that the leachates are not composed of a large component of residual salt solution trapped in the coalescer mesh. The coalescer was drained well before sending to SRNL.

Third, the results show that that most analytes do not show a time-based trend. As the contact time increases most analytes do not show a commensurate increase in concentration. There are some exceptions to this claim, such as the phosphorus values, but the increase over time in these cases is still relatively small. This behavior suggests any solids present are not difficult to dissolve nor are they packed deeply into the coalescer media.

Fourth, the concentrations of the analytes indicate that the amount of solids present on the coalescer is fairly small.

Fifth, while SRNL has leached and analyzed coalescer elements ${ }^{4,5}$ and parts of the Actinide Removal Process guard filter before, ${ }^{6}$ the current results show that far more titanium in the leaching samples that can be from the feed material. Considering there is less-than detectable amounts of $\mathrm{Ti}$ in the feed material, the titanium must be from monosodium titanate (MST). The MST fines must be passing through the filters at ARP and ending up caught on the pre-filters and coalescer, or the MST is dissolving at ARP, and precipitating in the MCU system to get caught on the pre-filters and coalescer. Other work from SRNL ${ }^{7}$ studying the ability of caustic solutions to dissolve MST solids shows that caustic leaching does occur, which makes titanium available for later precipitation and retention on the coalescer.

3.3 XRD Analysis When the coalescer arrived, a sample cut from the coalescer was analyzed by XRD. This method identifies types of crystalline or semi-crystalline solids in the sample. See Figure 1. 


\section{Figure 1. XRD Spectra for the Coalescer Sample}

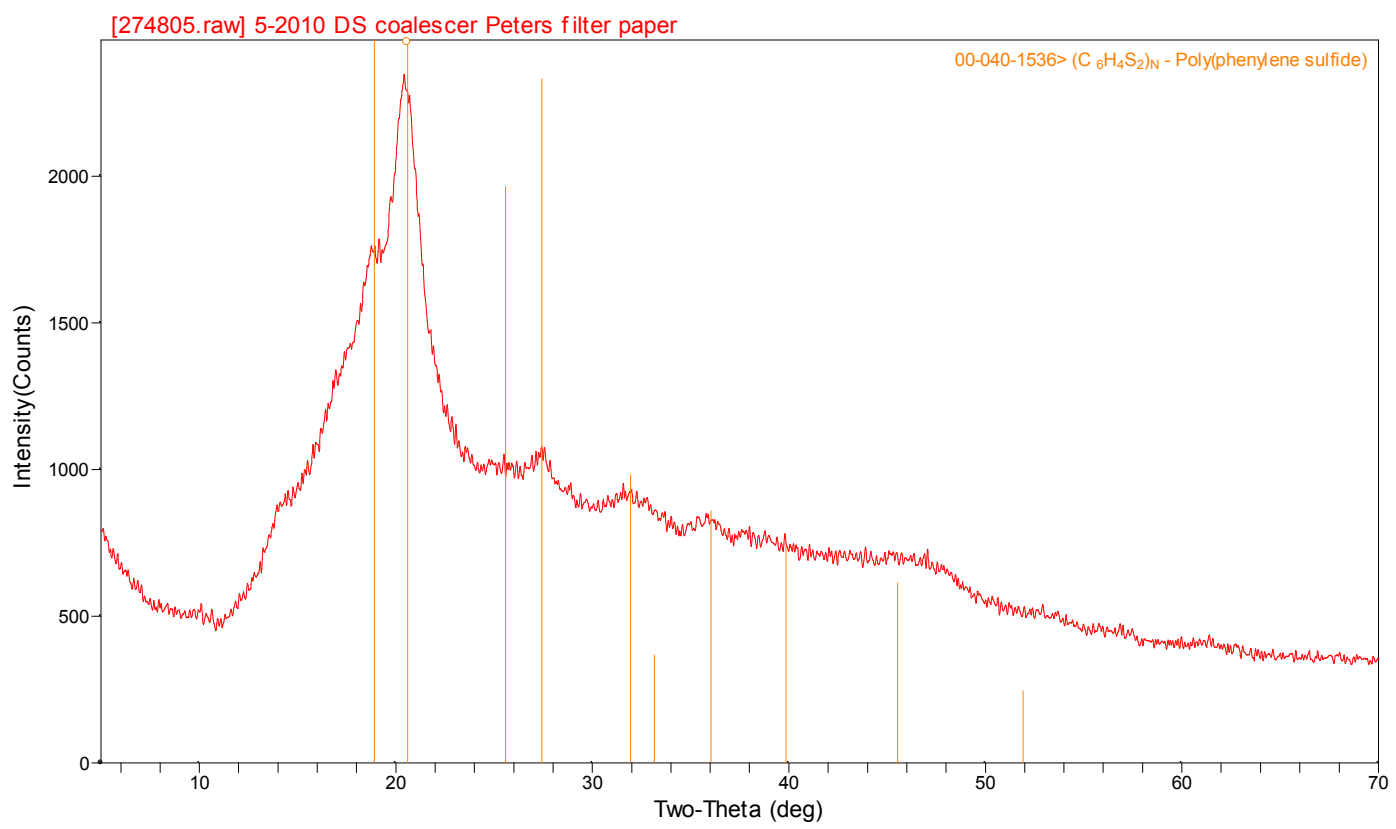

The spectrum only shows the coalescer media, with no evidence of any other crystalline solids being present. Given the low concentrations of materials leached from the coalescer slices, this result is not surprising. While there is no indication of titanium compounds from the XRD data, this is due to the difficulty in seeing amorphous MSTtype Ti-oxides in this analytical method.

\subsection{Conclusions}

The DSS coalescer was brought to SRNL and analyzed for evidence of fouling. Both the leaching studies and the XRD analysis indicate very little evidence of fouling via excessive solids. The presence of titanium is attributed to leaching of MST at ARP, followed by precipitation and retention on the DSS coalescer. 


\subsection{References}

${ }^{1}$ T. B. Peters, F. F. Fondeur, S. D. Fink, "Results from Analysis of the First and Second Strip Effluent Coalescer Elements from Radioactive Operations of the Modular Caustic-side Solvent Extraction Unit", SRNL-STI-2010-00088, June 2011.

${ }^{2}$ T. B. Peters, "ISDP3", SRNL-NB-2012-00022, February 9, 2012.

${ }^{3}$ S. E. Campbell, "Blend Evaluation for Tank 49H Feed for ISDP Salt Batch 3", X-ESR-H-00209, March 15, 2010.

${ }^{4}$ Peters, T. B., Fondeur, F. F. , Fink, S. D.," Diagnostic Analyses of the Decontaminated Salt Solution Coalescers from Initial Radiological Operations of the Modular Caustic-side Solvent Extraction Unit", SRNL-STI-2008-00369, Rev. 0, October 12, 2008.

${ }^{5}$ Peters, T. B., Fondeur, F. F., Fink, S. D., "Results of Analyses of Macrobatch 3 Decontaminated Salt Solution (DSS) Coalescer and Pre-filters", SRNL-STI-2011-00513, June 2012.

${ }^{6}$ Peters, T. B., Fondeur, F. F., Fink, S. D., "Results from Analysis of Actinide Removal Process Guard Filter”, SRNL-STI-2009-00456, Rev. 1, January 27, 2010.

${ }^{7}$ K. M. L. Taylor-Pashow, F. F. Fondeur, S. D. Fink, "Leaching of Titanium from Monosodium Titanate (MST) and Modified MST (mMST)", SRNL-STI-2012-00237, August 2012. 


\section{Distribution:}
S. D. Fink, 773-A
K. M. Fox, 999-W
C. C. Herman, 999-W
S. L. Marra, 773-A
F. M. Pennebaker, 773-42A
M. T. Keefer, 241-156H
E. A. Brass, 249-8H
D. J. Martin, 241-152H
B. A. Gifford, 704-56H
Q. L. Nguyen, 241-152H
T. B. Peters, 773-42A
C. A. Nash, 773-42A
F. F. Fondeur, 773-A
P. R. Jackson, 703-46A 